\title{
Le moustique Culiseta alaskaensis alaskaensis (Diptères Culicidae) en Dombes
}

\author{
par E. ROMAN et J. PICHOT \\ Laboratoire de Parasitologie, Université Claude-Bernard, \\ 8, avenue Rockefeller, F 69008 Lyon
}

\section{Résumé.}

Trois gîtes larvaires de Culiseta alaskaensis alaskaensis (Ludlow) ont été trouvés dans deux forêts des Dombes, au sud de Bourg. Ces stations sont, en Europe centrale et occidentale, parmi les plus méridionales de ce Moustique circumboréal.

\section{Summary.}

The mosquito Culiseta alaskaensis alaskaensis (Diptera Culicidae) in Dombes.

Three sites of Culiseta alaskaensis alaskaensis (Ludlow) were found in two forests of Dombes in the South of Bourg. These sites of circumboreal mosquito are among the most meridional ones in central and occidental Europe.

Nous jugeons intéressant de faire connaître la présence d'un Culicidae, peu signalé de France, sur le plateau des Dombes, au sud-ouest du département de l'Ain.

Parmi les Culiseta s. str., nous rapportons nos exemplaires provenant de cette région à l'espèce $C$. alaskaensis (Ludlow, 1906), qui ressemble beaucoup à $C$. annulata (Schrank) et à $C$. subochrea (Edw.).

Comme l'ont indiqué divers auteurs, nos adultes se distinguent extérieurement des imagos de ces Culiseta par l'absence d'anneaux blancs autour des fémurs et au milieu 
des protarses (fig. 1). D'après la monographie de E. Martini (1931), nous les déterminons $C$. $a$. alaskaensis et non $C$. a. indica (Edw.) [= wassilievi (Shingarew)], parce que le revêtement du dessus de la trompe et, aux ailes, celui des nervures les plus antérieures, ne sont formés que d'écailles sombres.
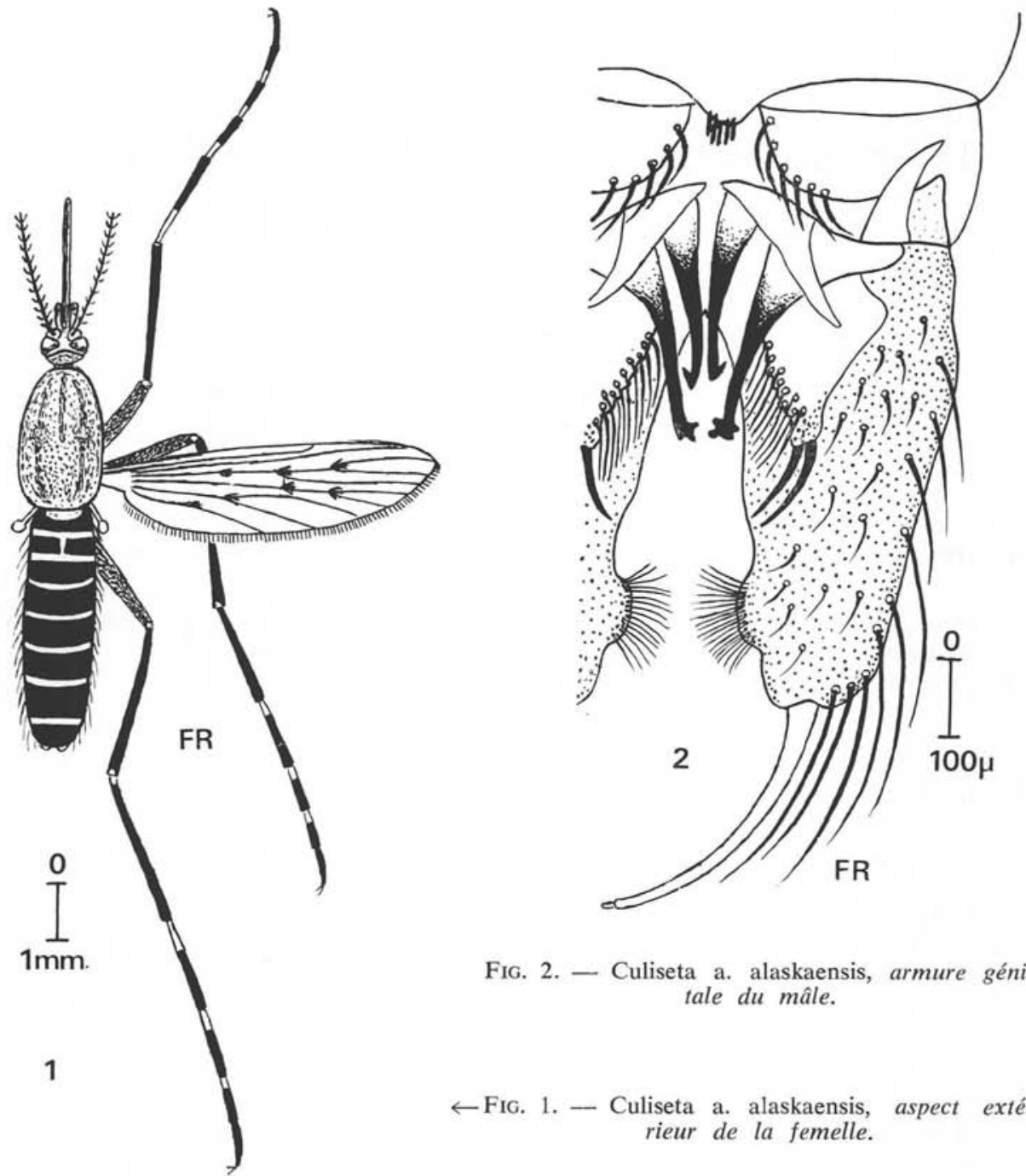

FIG. 2. - Culiseta a. alaskaensis, armure génitale du mâle.

$\leftarrow$ Fig. 1. - Culiseta a. alaskaensis, aspect extérieur de la femelle.

L'armure génitale du seul mâle obtenu, qui, d'abord introduite dans du lactophénol, s'est détériorée ensuite lors du montage dans le baume, présentait, ce qui la distingue de celle des Culiseta voisins, les caractères communs aux deux sous-espèces de $C$. alaskaensis, c'est-à-dire (nomenclature de F. W. Edwards, 1920) la présence sur 
chaque coxite d'une forte saillie préapicale, portant un bouquet de soies minces et assez longues, et, au sommet du lobe du $8^{\circ}$ tergite, d'une série de soies raides particulièrement rapprochées (fig. 2).

Nous avons déterminé l'exuvie d'une de nos larves $C$. alaskaensis, parce qu'elle présente, à notre avis, un caractère très net de ce Culiseta, autrefois indiqué par E. Martini, puis figuré par G. Sénevet et L. Andarelli (1959): sous le $9^{\circ}$ segment abdominal, il y a quatre touffes de soies en avant de l'aire barrée (nomenclature des auteurs français récents), alors qu'il n'y en a que deux chez $C$. annulata et chez C. subochrea.

Nous avons trouvé $C$. a. alaskaensis à l'état larvaire au sud de Bourg, dans deux forêts de feuillus, en des endroits à sol naturellement presque uni et sans revêtement de sphaignes. Nous avons reconnu trois gîtes, tous trois de faible profondeur (10 à $20 \mathrm{~cm}$ ), de largeur restreinte (entre $50 \mathrm{~cm}$ et $1 \mathrm{~m}$ ), mais d'assez grande longueur (50 à $100 \mathrm{~m}$ ), dans des fossés remplis par les pluies printanières, bordant des chemins d'exploitation de bois. Le 14 juin 1972, deux de ces gîtes ont été observés en forêt de Seillon: l'un, ombragé par des chênes et des charmes, ne renfermait pas de stades jeunes d'autres Culicidae; l'autre, en futaie de chênes, mais assez ensoleillé, avec des gros troncs empilés dans sa déclivité, donnait aussi asile à Culex pipiens $\mathrm{L}$., à $C$. hortensis Ficalbi et à Culiseta annulata; notre troisième gîte de C. a. alaskaensis, celui-ci, en forêt de la Réna, était une collection d'eau en pleine ombre avec aux alentours des aulnes, des saules-marsaults, des bouleaux et des charmes; il hébergeait en outre des larves d'Aedes cinereus Meig. et de A. sticticus (Meig.). Quelques adultes de C. $a$. alaskaensis ont éclos au Laboratoire. Le $\mathrm{pH}$ de l'eau de ces gîtes était de 7,0 pour le premier, de 6,4 pour le second et de 7,3 pour le troisième. Tous trois étant à sec ou à peu près en juin de l'année suivante, le Culiseta, objet de cette note, n'a pas été revu en Dombes en 1973.

Comme environnement, ces gîtes sont, vu l'absence de sphaignes, assez différents de ceux observés en Allemagne par F. Peus (1929) et par W. Mohrig (1969) ; le troisième est celui qui s'en rapproche le plus, en raison de la présence d'aulnes à son voisinage. Au point de vue concentration en ions hydrogène, nos évaluations ne sont pas bien différentes de celles ( $\mathrm{pH} 6,5$ et 6,9) de L. R. Natvig (1948) en Scandinavie ; contrairement à ses indications, l'eau de notre dernier gîte était légèrement acide.

C. a. alaskaensis affectionnant les climats froids, sa coexistence avec Culex hortensis, du bassin méditerranéen et d'Europe centrale, méritait une mention particulière.

Nous n'avons pas été piqués, dans les forêts des Dombes, par C. a. alaskaensis, comme l'ont mentionné en Allemagne F. Peus et E. Martini. En Dombes, nous ne l'avons jusqu'à présent observé qu'au printemps et au début de l'été. E. Noeldner (1953) note qu'en Alsace il n'a qu'une génération par an ; mais plus à l'est W. Mohrig en compte plusieurs et indique que son développement n'est interrompu que par le froid.

La sous-espèce nominale de Culiseta alaskaensis est circumpolaire. Au nord de l'Europe, elle est connue de Scandinavie, de Grande-Bretagne, de Pologne, de Tchécoslovaquie, d'Allemagne. En France, J. Rageau, J. Mouchet et E. Abonnenc (1970) ne la citent que d'Alsace d'après E. Noeldner. Elle se rencontre en Russie boréale, 
mais plus au sud de cette république, les documents à notre disposition ne nous permettent pas de délimiter les aires de répartition de $C$. a. alaskaensis et de $C$. $a$. indica. A. V. Maslov (1964) indique que cette dernière sous-espèce se trouve dans le centre et le midi de l'Europe ; il se peut que cette référence ne concerne que l'U.R.S.S. Car plus à l'ouest, les citations les moins septentrionales sont rapportées, sans mention de sous-espèce, ce qui semble signifier qu'il s'agit de $C$. alaskaensis type. C'est en tout cas lui qui, sans autre précision, est mentionné par E. Martini d'Autriche, où ce Moustique a été retrouvé à Eisenstein près du lac de Neusiedl (H. Aspök, 1966, H. Aspök et Ch. Kunz, 1966). Entre-temps, F. Mihályi (1959) a signalé des captures de C. alaskaensis en Hongrie, notamment sur les rives du lac Balaton, mais aussi plus au sud à Pecs. Cette localité et celles au nord-est de Lyon, voisines du $46^{\circ}$ degré de latitude nord, sont, en Europe centrale et occidentale, les plus méridionales de cette espèce.

Pour la rédaction de cette note, nous avons été efficacement aidés par nos collègues Mihályi, de Budapest, et Dorgelo, de l'Entente de démoustication de la région Rhône-Alpes. Nous leur adressons nos bien vifs remerciements.

\section{Bibliographie}

AsPöK (H.), 1966. - Parasitierung eines in Freiland aufgefundenen Intersexes von Aedes (Ochlerotatus) communis de Geer (Insecta, Culicidae) durch einen Pilz der Ordnung Blastocladiales. Zeits. Morphol. u. Okol. d. Tiere, 57, (3), 231-243.

- et KunZ (Ch.), 1966. - Isolierung des Tahynavirus an Stechmücken in Osterreich. Arch. f. Virusforsch, 17 (1), 7-15.

EDWARDS (F.W.), 1920. - The nomenclature of the parts of the male hypopygium of Diptera Nematocera with special reference to mosquitoes. Ann. trop. Med. and Parasitol., 14 (1), 23-40.

MARTINI (E.), 1931. - Culicidae in Lindner, die Fliegen der paläarktischen Region, E. Schweizerhart'sche Verlag, Stuttgart.

Maslov (A. V.), 1964. - Systématique des Moustiques suceurs de sang du groupe Culiseta (Dipt. Culicidae). Entom. obozr., 43, 193-217 (en Russe).

Mihalyi (F.), 1959. - Revision der aus dem Karpatenbecken stammenden Stechmücken der ungarischen Dipterensammlungen. Folia entom. Hung., ser. nov., 12, 139-162.

Mohrig (W.), 1969. - Die Culiciden Deutschland. Gustav Fischer, Verlag, Iena.

Natvig (L. R.), 1968. - Contribution to the knowledge of the danish and fenoscandian Mosquitoes Culicini. Suppl. Norsk. entom. Tidsk., Oslo.

Noeldner (E.), 1953. - Moustiques rares ou peu connus d'Alsace. Diptera, 11, 5-45.

Peus (F.), 1929. - Beiträge zur Faunistik und Ökologie der einheimischen Culiciden, I. Zeits. f. Desinfekt., 21 (3), 76-81.

Rageau (J.), Mouchet (J.) et Abonnenc (E.), 1970. - Répartition géographique des Moustiques (Diptera Culicidae) en France. Cah. O.R.S.T.O.M., sér. Entom. méd. Parasitol., 8 (3), 289-316

Sevenet (G.), et Andarelli (L.), 1959. - Les Moustiques de l'Afrique du Nord et du bassin méditerranéen. Encycl. entom., 37, Lechevalier, édit., Paris. 